\section{Band 19, Heft 1, März 2009}

Editorial

4 Internetgestiitzte Therapieprogramme:

Gefahr für die traditionelle Psychotherapie?

Hohagen, F. (Lübeck)

Übersichtsarbeit

6 Eine kognitive Perspektive auf die Insomnie:

Theorie und Behandlung

Birrer, E. (Luzern); Junghanns-Royack, K. (Bremerton, WA);

Heidenreich, T. (Esslingen)

\section{Originalarbeit - Diagnostik}

14 Figurunzufriedenheit von Frauen in Abhängigkeit vom Lebensalter: Normative Daten für den Fragebogen zum Figurbewusstsein

Pook, M. (München); Brähler, E. (Leipzig);

Tuschen-Caffier, B. (Freiburg i.Br.)

Kurzbericht - Kinder und Jugendliche

22 Typische klinische Problemkonstellationen bei Patienten der Psychologischen Kinderambulanz der Universität Bremen

Jacobs, C.; Tischler, L; Petermann, F. (Bremen)

Für die Praxis

28 Reduktion von Prokrastination: Module zum pünktlichen Beginnen und realistischen Planen Höcker, A.; Engberding, M.; Beißner, J.; Rist, F. (Münster)

33 Wann sind Sorgen pathologisch?

Hoyer, J.; Heidrich, S. (Dresden)

40 Modellvorschlag für die kognitive Verhaltenstherapie der Kleptomanie

Bohne, A. (Münster); Stevens, S. (Giessen)

Interview

47 Peter Weiden: «Viele psychosoziale Interventionen für Schizophrenie akzeptieren die fehlende Krankheitseinsicht der Patienten nicht» Lencer, R. (Lübeck)

\section{Vol. 19, Issue 1, March 2009}

Editorial

4 Internet-Based Therapeutic Programs:

A Challenge for Conventional Psychotherapy?

Hohagen, F. (Lübeck)

Review Article

6 A Cognitive Perspective on Insomnia:

Theory and Treatment

Birrer, E. (Luzern); Junghanns-Royack, K. (Bremerton, WA);

Heidenreich, T. (Esslingen)

Original Article - Diagnostics

14 Female Body Dissatisfaction across Different Age Groups: Normative Data for the German Version of the Body Shape Questionnaire Pook, M. (München); Brähler, E. (Leipzig); Tuschen-Caffier, B. (Freiburg i.Br.)

Short Report - Children and Adolescents

22 Typical Clinical Problem Patterns in Patients of the Psychological Children's Clinic at the University of Bremen

Jacobs, C.; Tischler, L; Petermann, F. (Bremen)

For the Practitioner

28 Reduction of Procrastination: Working Steps Aiming at Punctuality and Realistic Planning

Höcker, A.; Engberding, M.; Beißner, J.; Rist, F. (Münster)

33 When Are Worries Pathological?

Hoyer, J.; Heidrich, S. (Dresden)

40 Treatment Model for Cognitive Behavior Therapy of Kleptomania

Bohne, A. (Münster); Stevens, S. (Giessen)

Interview

47 Peter Weiden: 'Many Psychosocial Interventions for Patients with Schizophrenia Do Not Acknowledge These Patients' Lack of Insight into Their Illness' Lencer, R. (Lübeck) (c) 2009 S. Karger GmbH, Freiburg

Fax +49761 452071

Information@Karger.de

www.karger.com 


\section{Verhaltenstherapie}

\section{Band 19, Heft 1, März 2009}

Pro und Contra

53 Macht Selbsterfahrung bessere Therapeuten? Rief, W. (Marburg); Freyberger; H.J. (Greifswald) Sartory, G. (Wuppertal)

58 Buchbesprechungen

62 Fort- und Weiterbildung

67 Mitteilungen der Verbände

69 Tagungen und Kongresse

56 Impressum

71 Hinweise für Autoren

U2 Erläuterungen zum Titelbild (2. Umschlagseite) Leibl, C.; Veh, C.; Wagner, J. (Prien am Chiemsee)

\section{Vol. 19, Issue 1, March 2009}

Pro and Con

53 Does Self-Awarenesse Make Better Therapists? Rief, W. (Marburg); Freyberger; H.J. (Greifswald); Sartory, G. (Wuppertal)

58 Book Reviews

62 Education

67 Information by Behavior Therapy Associations

69 Meetings and Conferences

56 Imprint

71 Guidelines for Athors

C2 Explanations on the Title (Inside front cover) Leibl, C.; Veh, C.; Wagner, J. (Prien am Chiemsee)
Einen Ausblick auf den Inhalt der kommenden Hefte finden Sie auf Seite 72 .
Forthcoming papers are listed on page 72.

\section{KARGER}

Fax +497614520714 